\title{
Real-World Data as an Evidence Source in Nuclear Medicine
}

\author{
Ariel B. Bourla ${ }^{1}$ and Ken Herrmann ${ }^{2-4}$ \\ ${ }^{1}$ Flatiron Health, Inc., New York, New York; ${ }^{2}$ Department of Nuclear Medicine, University Hospital Essen, Essen, Germany; ${ }^{3}$ German \\ Cancer Consortium, University Hospital Essen, University of Duisburg-Essen, Essen, Germany; and ${ }^{4}$ West German Cancer Center, \\ Essen, Germany
}

$\mathbf{T}$ hroughout the course of routine clinical practice, real-world data are obtained from various sources, including medical claims, product and disease registries, electronic health records (EHRs), and prospective observational study settings. Real-world evidence (RWE) is generated by analyzing real-world data for specific research questions to describe patients, treatments, and outcomes (1). RWE use is becoming particularly relevant in oncology, as only a small percentage of adult cancer patients worldwide are enrolled in clinical trials whereas the number of therapeutic options and the segmentation of patient populations are rapidly increasing, leading to the explosive growth of research questions and evidence gaps (2).

RWE holds the promise of expanding evidence generation into routine clinical care settings to address questions that cannot be answered with data from traditional clinical trials, such as those related to rare cohorts of patients or those for which there is no clinical equipoise to permit ethical randomization. In drug development, RWE can be used to inform clinical trial design and feasibility during clinical development and to provide natural history and standard-of-care context for the study population of interest. After drug approval, RWE can support regulators and health technology assessment bodies by providing real-world information on safety, effectiveness, and cost effectiveness in the context of postmarketing requirements (3). Finally, RWE can enable precision medicine by shedding light on real-world adoption patterns for new therapies and diagnostics, and by comparing effectiveness between different therapies in targeted populations (4).

Although many RWE initiatives rely on costly manual data input or already structured information, the broad adoption of EHRs worldwide over the past decade represents a promising source of rich clinical data. Deriving meaningful and trustworthy insights from the data, however, requires standardized data processing, rigorous quality assurance, and application of sophisticated analytic approaches. Harmonizing structured data, such as diagnosis codes, medication administrations, and laboratory results, to a common data model enables the merging of data from different sources. Extracting information from unstructured documents, such as free-text clinical notes or radiology and biomarker reports, allows for collection of deeper, more nuanced data elements such as assessments of tumor burden and response. Although advances in artificial intelligence hold the promise for automated interpretation of such unstructured

Received Oct. 15, 2020; revision accepted Nov. 2, 2020.

For correspondence and reprints contact: Ken Herrmann, Department of Nuclear Medicine, University Hospital Essen, Hufelandstrasse 55, Essen, 45147 Germany.

E-mail: ken2404@web.de

Published online Nov. 27, 2020.

COPYRIGHT (c) 2021 by the Society of Nuclear Medicine and Molecular Imaging. DOI: 10.2967/jnumed.120.258665 data, high-quality evidence generation currently still relies on trained human curators' ability to follow standardized policies and procedures to guarantee the internal consistency of the evidence $(4,5)$.

For RWE to generate actionable insights, it must provide information on clinical outcomes. In oncology, attaining this information requires new approaches to capture tumor burden assessments that ought to be tested for reliability-for example, to capture real-world progression and real-world response from unstructured EHR documents as an alternative to RECIST (6). In addition, information on survival can be collected from a combination of mortality surveillance tools beyond information in the EHR, such as national databases.

If RWE is deemed appropriate for a relevant scenario, a specific data source must be determined as fit for the purpose of answering the underlying research question. To determine whether a dataset is regulatory-grade, Miksad and Abernethy proposed a checklist that defines attributes including quality, completeness, transparency, generalizability, timeliness, and scalability (5). Based on a specific RWE source, a study design should be developed to address these attributes, including an analysis of the data source and target population (e.g., geographic representation), statistical approaches such as propensity score methods to control for measured confounding, and a detailed statistical analysis plan (7). Such a careful and transparent design is critical in leveraging RWE for decision making along the product life cycle. Ongoing research into transparent and standardized approaches for RWE analyses remains critical. Organizations such as the Duke-Margolis Center for Health Policy are developing important guidance for the application of RWE (3). Franklin et al. proposed a similar structured process for using RWE for regulatory decision making (7).

Several recent examples demonstrate that EHR-derived RWE already has the ability to impact access to new treatments and hence clinical practice in specific settings. In one example, the U.S. Food and Drug Administration approved a supplemental new drug application for palbociclib (Ibrance; Pfizer) to expand the label to male patients with breast cancer. Palbociblib had previously been approved for use in women with metastatic breast cancer. RWE derived from EHRs and other sources were used in addition to past clinical trial data to evaluate effectiveness and safety for the label expansion to male patients (8). In another example, the Pharmacovigilance Risk Assessment Committee of the European Medicines Agency accepted RWE as part of a postapproval risk management plan for trastuzumab emtansine (Kadcyla; Genentech). EHR-derived data were used to evaluate the safety of the product in metastatic breast cancer patients with a low left ventricular ejection fraction before initiation of treatment, a patient group that was excluded from clinical studies (9). 
These are encouraging examples of the use of RWE to describe and facilitate the availability of treatments for patients with rare clinical conditions or those excluded from clinical trials in which lengthy and costly prospective clinical studies may not be feasible. RWE also has significant potential applications in understanding the value of diagnostic or therapeutic radiologic procedures. Nuclear medicine procedures that are in common use may lack extensive prospective clinical evidence before becoming available, resulting in a heterogeneous reimbursement landscape and uncertainty in medical decision making. Collection and analysis of routinely collected RWE regarding the use and performance of novel diagnostic or therapeutic radiology could potentially generate the evidence needed to further assess the value of nuclear medicine procedures in specific clinical contexts.

For example, diagnostic PET procedures are sometimes performed before (and often even without) reimbursement. Whereas ${ }^{18}$ F-FDG PET for melanoma is typically covered by insurance plans in the United States, the lack of prospective clinical studies demonstrating survival benefit limits reimbursement in Germany. Analysis of RWE could potentially demonstrate whether there is added value for ${ }^{18}$ F-FDG PET by comparing patient groups managed with and without access to ${ }^{18}$ F-FDG PET. Another opportunity might be to understand the therapeutic effectiveness of nuclear medicine treatments such as ${ }^{89} \mathrm{Sr},{ }^{153} \mathrm{Sm}$, and ${ }^{131} \mathrm{I}$ that were not subject to large-scale prospective randomized trials. Collection and analysis of real-world data may be able to support the effectiveness of these therapies. Of course, as in any observational study, appropriate analytic methodology is necessary to mitigate potential unmeasured confounders and biases (10).

High-quality RWE from EHRs is increasingly recognized as an opportunity to fill evidence gaps, especially for applications for which prospective clinical studies are infeasible or when the potential benefit of the diagnostic or therapeutic intervention is large, as suggested by the U.S. Food and Drug Administration RWE program (11). Nuclear medicine technology, in both diagnostics and therapeutics, represents a remaining frontier that could benefit from new insights derived from RWE.

\section{DISCLOSURE}

No potential conflict of interest relevant to this article was reported.

\section{REFERENCES}

1. Real-world evidence. U.S. Food and Drug Administration website. https:// www.fda.gov/science-research/science-and-research-special-topics/real-world-evidence. Accessed November 17, 2020.

2. Lee SJC, Murphy CC, Geiger AM, et al. Conceptual model for accrual to cancer clinical trials. J Clin Oncol. 2019;37:1993-1996.

3. Adding real-world evidence to a totality of evidence approach for evaluating marketed product effectiveness. Duke Margolis Center for Public Policy website. https://healthpolicy.duke.edu/publications/adding-real-world-evidence-totality-evidenceapproach-evaluating-marketed-product. Published December 19, 2019. Accessed November 17, 2020.

4. Di Maio M, Perrone F, Conte P. Real-world evidence in oncology: opportunities and limitations. Oncologist. 2020;25:e746-e752.

5. Miksad RA, Abernethy AP. Harnessing the power of real-world evidence (RWE): a checklist to ensure regulatory-grade data quality. Clin Pharmacol Ther. 2018; 103:202-205.

6. Arbour KC, Luu AT, Luo J, et al. Deep learning to estimate RECIST in patients with NSCLC treated with PD-1 blockade. Cancer Discov. September 21, 2020 [Epub ahead of print].

7. Franklin JM, Glynn RJ, Martin D, Schneeweiss S. Evaluating the use of nonrandomized real world data analyses for regulatory decision making. Clin Pharmacol Ther. 2019;105:867-877.

8. Wedam S, Fashoyin-Aje L, Bloomquist E, et al. FDA approval summary: palbociclib for male patients with metastatic breast cancer. Clin Cancer Res. 2020;26:1208-1212.

9. EUPAS20684. European Network of Centres for Pharmacoepidemiology and Pharmacovigilance website. http://www.encepp.eu/encepp/viewResource.htm? id $=22158$. Published August 22, 2017. Updated December 12, 2019. Accessed November 17, 2020.

10. Bartlett VL, Dhruva SS, Shah ND, Ryan P, Ross JS. Feasibility of using real-world data to replicate clinical trial evidence. JAMA Netw Open. 2019;2:e1912869.

11. Framework for real-world evidence program. U.S. Food and Drug Administration website. https://www.fda.gov/media/120060/download. Published December 2018. Accessed November 17, 2020. 